\title{
Risk prediction for death and end-stage renal disease does not parallel the real-life trajectory of older patients with advanced chronic kidney disease - a Romanian center experience.
}

Type

Research paper

\section{Keywords}

Mortality, dialysis, Bansal score, KFRE

\begin{abstract}
Introduction

In 2016, the European Renal Best Practice (ERBP) group published a guideline on the management of older adults with advanced chronic kidney disease (CKD). Two risk scores were highlighted: Bansal score for mortality, and Kidney Failure Risk Equation (KFRE) for estimating progression to end-stage kidney disease (ESRD). Our group, as part of the ERBP team, aimed to apply these risk prediction tools in a cohort of older adults with eGFR $<45 \mathrm{ml} / \mathrm{min} / 1.73 \mathrm{~m} 2$.
\end{abstract}

Material and methods

This retrospective study included adults aged $\geq 65$ years with CKD stage $3 \mathrm{~b}-4$, evaluated at a Romanian Outpatient Nephrology Department between October 2016 - October 2018. Bansal score was calculated for all subjects and then KFRE was used in the low mortality risk group. Outcomes were death or reaching ESRD. These outcomes were used to compare the difference between the estimated trajectory and real-life trajectory of patients. They were followed up until September 2019.

Results

From the total population ( $\mathrm{N}=958$ patients), more than half $(\mathrm{N} 1=548,57.2 \%)$ had a high mortality risk. In the remaining group with low mortality risk ( $\mathrm{N} 2=410,42.8 \%)$, a significant percentage $(75.4 \%)$ presented a low risk of progression to ESRD.

Real-life events consisted of 164 deaths and 31 dialysis initiations. We found similar death rates in the two groups (high versus low risk of mortality). There was no difference in the rate of dialysis initiation between subjects with a high or low risk of progression to ESRD.

\section{Conclusions}

Risk prognostication for death and ESRD did not parallel the real-life trajectory of our older patients with advanced CKD. 


\section{ABSTRACT \\ Background}

In 2016, the European Renal Best Practice (ERBP) group published a guideline on the management of older adults with advanced chronic kidney disease (CKD). Two risk scores were highlighted: Bansal score for mortality, and Kidney Failure Risk Equation (KFRE) for estimating progression to end-stage kidney disease (ESRD). Our group, as part of the ERBP team, aimed to apply these risk prediction tools in a cohort of older adults with eGFR $<45 \mathrm{ml} / \mathrm{min} / 1.73 \mathrm{~m}^{2}$.

\section{Methods}

This retrospective study included adults aged $\geq 65$ years with CKD stage $3 b-4$, evaluated at a Romanian Outpatient Nephrology Department between October 2016 - October 2018. Bansal score was calculated for all subjects and then KFRE was used in the low mortality risk group. Outcomes were death or reaching ESRD. These outcomes were used to compare the difference between the estimated trajectory and real-life trajectory of patients. They were followed up until September 2019.

\section{Results}

From the total population ( $\mathrm{N}=958$ patients), more than half $(\mathrm{N} 1=548,57.2 \%)$ had a high mortality risk. In the remaining group with low mortality risk $(\mathrm{N} 2=410,42.8 \%)$, a significant percentage $(75.4 \%)$ presented a low risk of progression to ESRD.

Real-life events consisted of 164 deaths and 31 dialysis initiations. We found similar death rates in the two groups (high versus low risk of mortality). There was no difference in the rate of dialysis initiation between subjects with a high or low risk of progression to ESRD.

\section{Conclusions}

Risk prognostication for death and ESRD did not parallel the real-life trajectory of our older patients with advanced CKD.

KEYWORDS: Bansal score, Dialysis, KFRE, Mortality. 


\section{INTRODUCTION AND AIMS}

The increasing number of adults surviving to older ages has led to increasing incidence and prevalence of those diseases common among older adults, including chronic kidney disease (CKD) $[1,2]$. More importantly, the renal disease in geriatric patients rarely occurs as a sole condition, being frequently superimposed to other diseases that contribute to CKD development, including diabetes or cardiovascular (CV) disease [3, 4]. This becomes more problematic in the presence of advanced CKD, corresponding to an estimated glomerular filtration rate (eGFR) below 45 $\mathrm{ml} / \mathrm{min} / 1.73 \mathrm{~m}^{2}$, where the high burden of comorbidities contributes to the high degree of frailty [5], decreased functional status, decreased quality of life, increased risk of hospitalization or even death [6-8]. Therefore, it is unsurprising that CKD, a growing condition in older patients, will represent a challenge in terms of appropriate management and also healthcare costs.

The real-life management of older patients with advanced CKD is often challenged by the competing risk of ESRD or death before developing ESRD. Thus, early referral to a nephrologist and preparation for kidney replacement therapy may become unnecessary and potentially harmful for those patients whose risk of death is higher than the risk of ESRD. Even if, prior studies suggest that the risk of death is higher than the risk of ESRD, in clinical practice, identifying the risk in individual patients remains difficult due to the scarcity of studies that include older subjects [9].

From this point of view, in 2016, European Renal Best Practice (ERBP) group published a clinical practice guideline addressing the clinical approach to patients aged $>65$ years with an eGFR $<45$ $\mathrm{ml} / \mathrm{min}$ per $1.73 \mathrm{~m} 2$ [10]; the risk stratification concept which impacts therapeutic decisions was underlined by this guideline - for example, whether renal replacement therapy (RRT) should be initiated or conservative treatment be maintained. Two risk stratifications scores were emphasized: Bansal score for prognosticating risk of death in the medium term, and Kidney Failure Risk Equation (KFRE) for estimating progression of CKD stage $3 \mathrm{~b}$ or 4 to ESRD, both equations being validated in CKD population [11, 12]. The algorithm from the guideline implies that a patient with a high Bansal score, corresponding to high mortality risk, will benefit from a conservative approach regarding ESRD risk, with focus on advanced care planning and not stressing the future need for RRT. The same direction of management is suggested for the elderly with low mortality risk, but with a frailty state. On the other hand, if the patient has low mortality risk, is not frail and 
has a high risk for progression to ESRD, the guideline recommends maximized nephroprotection and pre-dialysis counselling, including modality selection.

Our group, as part of the ERBP team, aimed to evaluate a cohort of elderly patients with advanced CKD and apply a slightly different framework than the one proposed by the guideline, consisting of risk prediction for both mortality and progression to ESRD in the same population concurrently; after dividing the population into groups of mortality risk, we described their real-life trajectory in terms of either reaching ESRD/death. Furthermore, we are proposing a risk threshold for Bansal score since what constitutes a high risk for mortality outcome is not defined by the ERBP guideline.

\section{MATERIALS AND METHODS}

\section{Study design and participants}

We retrospectively evaluated a cohort of patients aged $\geq 65$ years with advanced CKD (eGFR $<45$ $\mathrm{ml} / \mathrm{min} / 1.73 \mathrm{~m}^{2}$ ) followed between October 2016 - October 2018 in the nephrology unit of a tertiary referral hospital from Iași, Romania (Dr C.I. Parhon University Hospital). These patients were considered to be a representative sample for the north-east population of Romania regarding various renal pathology.

\section{Definition of variables}

A set of demographic, clinical and biochemical variables were required to assess a patient's risk of mortality/progression to ESRD. These data were collected from the patient's electronic medical records. Glomerular filtration rate was estimated (eGFR) using the Chronic Kidney DiseaseEpidemiology Collaboration (CKD-EPI) formula [13] based on serum creatinine values.

\section{Outcomes}

After predicting individual risk of mortality and progression to ESRD, the entire cohort was divided into groups of risk. The follow-up period comprised the evaluation of their first future clinical outcome (death or reaching ESRD), which allowed comparison between estimated trajectory and real-life trajectory.

\section{Risk prediction for mortality and ESRD}


Individual risk for mortality was predicted using Bansal score, a nine-variable equation model developed in a US cohort of 828 participants aged $\geq 65$ years with an eGFR $<60 \mathrm{ml} / \mathrm{min}$ per 1.73 $\mathrm{m}^{2}$, from the Cardiovascular Health Study (CHS) [12]. The Bansal score was already validated in an independent cohort of 789 community-living elders with CKD from the Health, Aging and Body Composition Study [14], with the same inclusion criteria regarding age and eGFR.

Each of the 9 variables from the Bansal equation had a corresponding score and the total score was associated with an estimate of risk. A total score of 7 (associated with a mortality risk of 53.82\%) was established as a cut-off value to differentiate between 2 groups: high risk of mortality (Bansal $\geq 7$ ) and low risk of mortality (Bansal $<7$ ), given the fact that the ERBP guidelines don't define a threshold for high risk in respect to mortality outcome.

For estimating the risk for progression to ESRD at 5 years, we used the 4-variable Kidney Failure Risk Equation (KFRE), according to the algorithm proposed by the ERBP guideline [10]. This was calculated in the low mortality risk group using the KFRE equation which was initially developed and validated in 2 Canadian CKD cohorts [11], but it was later validated in 31 multinational cohorts including more than 721,000 participants with GFR $<60 \mathrm{ml} / \mathrm{min} / 1.73 \mathrm{~m}^{2}$ [15].

\section{Statistical analysis}

Continuous variables were expressed as mean \pm standard deviation (SD), while the categorical ones as a percentage of frequency. For the categorical variables, the between-group comparisons were performed using the $\chi 2$ test.

A P-value $<0.05$ was considered to be statistically significant. All statistical analyses were performed with SPSS 21.0 (SPSS, Chicago, IL).

\section{RESULTS}

A total of 1047 patients who fulfilled the including criteria (age $\geq 65$ years and eGFR $<45$ $\mathrm{ml} / \mathrm{min} / 1.73 \mathrm{~m}^{2}$ ) were evaluated in the above-mentioned period. Of those, 89 subjects were excluded as a result of missing data required for calculation of the risk scores. The final cohort included 958 patients. The mean age was $74.3 \pm 6.9$ years, and the gender distribution was similar (50.6\% female vs. $49.4 \%$ male). From the comorbid conditions that were components of Bansal 
score, we found a high prevalence of diabetes (47.4\%) in our elderly population, followed by heart failure (34\%) and stroke (11.2\%). Baseline characteristics of study participants by risk scores and by first future outcome (death or dialysis) are reported in Table I.

The predicted trajectory in terms of reaching ESRD / death

After applying the Bansal mortality score, we identified 548 patients (57.2\%) with high risk (N1 group) and 410 patients (42.8\%) with low risk of mortality (N2 group). The subjects from N1 group were more frequently male, had a more advanced age and a higher prevalence of comorbidities. Individual risk of progression to ESRD was then estimated in the N2 group, using 4-variable KFRE. Nearly a quarter of this group $(24.6 \%, 101$ subjects $=$ N2a subgroup $)$ presented a high risk of progression, with the remaining 309 subjects (N2b subgroup) having predicted low mortality and low risk of progression to ESRD.

The real-life trajectory in terms of reaching ESRD / death

The end date for follow-up was September 1, 2019. From the entire cohort, 31 patients (3.23\%) started renal replacement therapy and 164 patients $(17.11 \%)$ died as their first clinical event.

When comparing the two groups (deaths vs. dialysis) according to the baseline degree of comorbidity, patients who ultimately died had a higher percentage of diabetes (47\% vs. $41.9 \%$, nearly half of the group) and a similar prevalence of heart failure (28.1\% vs. $29.1 \%$, approximately one third of patients in each group). Stroke prevalence at baseline was higher in the subjects who ultimately entered chronic dialysis than in the ones who died (19.3 vs. $10.3 \%)$.

The RRT initiation rate was higher in the $\mathrm{N} 1$ group as compared with the N2 one, but it did not reach statistical significance ( $3.6 \%$ vs. $2.7 \%, \mathrm{p}=0.46$ ). Furthermore, when analysing only patients with a low risk for mortality, there was no difference in the rate of RRT initiation between subjects with a high or low risk of progression to ESRD ( $\mathrm{N} 2$ a group $-5.0 \%$ vs $\mathrm{N} 2 \mathrm{~b}$ group $-1.9 \%, \mathrm{p}=0.15$ ). The mortality percentage was not significantly different between the $\mathrm{N} 1$ and $\mathrm{N} 2$ groups (15.5\% vs. $19.3 \%$, respectively, $\mathrm{p}=0.14$ ). Similarly, when analysing only patients with a Bansal score below 7 , there was no difference in the death rates between the subjects with a high or low risk of progression to ESRD (N2a group - $18.8 \%$ vs N2b group - 19.4\%, $\mathrm{p}=1.00$ ). 
Flowchart 1 depicts the real-life trajectory of the study population in terms of reaching dialysis/death.

\section{DISCUSSION}

This is the first study who sought to see how the two risk prediction scores for mortality and progression to ESRD proposed by the ERBP guideline were implemented in a large population of elderly patients with advanced CKD from Eastern Europe and whether the predicted risk overlaps with real-life outcomes. One of the major findings was that more than half of our elderly subjects had an estimated 5-year risk of mortality that exceeded 50\%. This fact draws attention to a growing population of sick elderly patients because having such a high risk of death often involves having an accumulation of diseases like diabetes, heart failure or stroke, according to Bansal score. For this segment of multimorbid older patients with a relatively short life expectancy, efforts should be made to maximize functional status and quality of life. After all, maintaining independence is ranked as a top health outcome priority from the older patient's point of view, weighing more than the wish to stay alive, as a recent study shows [16].

The use of both equations proposed by the ERBP guideline was already validated in a cohort study of Norwegian elderly population with advanced CKD, both having overall good performance, with higher diagnostic accuracy for KFRE as compared to Bansal score [17, 18]. When quantifying how the risk of death and ESRD compare to one another, the authors found that mortality risk was much higher than ESRD risk (10:1), with only a small proportion of individuals $(2.6 \%)$ having a predicted risk of ESRD higher than their risk of death over five years.

In contrast, in our study, we went a step forward by proposing a threshold of 7 for mortality score that corresponds to an estimated risk of $53.82 \%$ and then calculating the risk of progression to ESRD only in those with low mortality risk. The observed mortality rate was approximately five times greater than the initiation of dialysis, supporting the idea that in the elderly population, the risk of death exceeds the risk of progression to ESRD, even among those with severe reductions in eGFR (e.g. $<30 \mathrm{ml} / \mathrm{min} / 1.73 \mathrm{~m}^{2}$ ) [19]. When we used the Bansal score to estimate mortality risk, we found that more than half of the population had a high risk. Nevertheless, the interesting finding was that the real-life death rates were similar in the two groups (N1 with a high risk of 
mortality versus N2 with low risk of mortality), suggesting the discrepancy between predicted versus real-life trajectory of the groups in terms of mortality.

From 31 patients who ultimately entered a chronic dialysis program, 20 subjects $(64,51 \%)$ had a high risk of mortality at baseline, derived implicitly from a higher degree of medical comorbidities (diabetes, heart failure, stroke). The large proportion of multimorbid patients with a high risk of mortality who entered dialysis is inconsistent with the ERBP guideline recommendation of not promoting dialysis in this context, outlining the difficulty of applying the guideline in real-life settings. Also, the similar dialysis initiation rates irrespective of kidney failure risk prediction shows that risk prognostication for ESRD did not parallel the real-life trajectory of our elderly patients with advanced CKD.

Estimating the risk of death and progression to ESRD in older adults is important because it gives the possibility of better guidance on the decision to start dialysis or to choose a conservative approach. In a first clinical scenario, a patient with a high risk of developing ESRD needs an early referral to a nephrologist, preparation for dialysis (modality selection, placement of an arteriovenous fistula) or transplant evaluation. The second scenario involves an elderly who has a greater risk of dying than experiencing ESRD, in which case planning dialysis comes with financial, psychological and physical costs that are unnecessary, making conservative care a more suitable option. Although conservative management provides reasonable symptom control and is likely to improve quality of life in elderly patients with CKD [20], only 1/3 of nephrologists discuss this approach with them, mainly due to the common view of conservative care as "no care" [21].

For the nephrologists, presenting conservative management as an option is challenging because this approach may be considered passive rather than active, although it encompasses a whole spectrum of services available to the patients (nutrition, psychology, social work, palliative care). The conservative approach is part of end-of-life care for older adults with advanced CKD, and is mandatory in an era of patient-centered care.

In our study, metabolic and cardiovascular pathology was a frequent characteristic of the population, with almost half of the subjects having diabetes and approximately one third having heart failure. This finding underlines the high burden of comorbidities in this Eastern European elderly cohort with advanced CKD, in contrast to other European populations; for example, these 
pathologies were found to a lesser extent in Hallan's recent Norwegian analysis of 1,188 subjects with same inclusion criteria regarding age and eGFR [17].

Our study has several strengths. We implemented risk stratification tools from ERBP guideline for the first time in a large sample of older patients with advanced CKD from Eastern Europe. We also defined a threshold for mortality score, since what constitutes a high risk in respect to mortality outcome is not mentioned in ERBP guideline. Comparing the estimated trajectory of our older patients (risk of mortality, risk of progression to ESRD) with real-life events is another important aspect, since the vulnerability of geriatric patients may predispose to unpredictable outcomes.

The fact that frailty could not be evaluated due to the retrospective design of the study is a limitation of our analysis. However, formal frailty scores are so heterogeneous that results based on different scoring systems cannot be compared or pooled [22]. We also studied only subjects that were evaluated at the Outpatient Department of Nephrology, which may limit the generalizability of our results. Another important issue is the limited power of the study, which is mirrored by the small number of certain events. For example, there were 31 events for initiation of RRT (11 in N2 group with low risk of mortality, distributed as follows: 5 in N2a subgroup with high risk of progression and 6 in N2b subgroup with low risk of progression to ESRD $\rightarrow$ Flowchart 1). These events were observed in a follow-up period of up to three years, while Bansal score and KFRE give an estimate of mortality and progression to ESRD over five years; this fact could explain the incongruence between predicted outcomes and real-life outcomes of our population.

In conclusion, although the two prediction scores proposed by ERBP guideline to estimate mortality and progression to ESRD are well-validated in previous studies [14, 15, 17], they did not overlap with real-life outcomes of our older patients with advanced CKD. Another possible explanation may be the different clinical profile of our older subjects, marked by a high burden of comorbidities, which should be a promotor for optimizing outcomes. 


\section{ACKNOWLEDGEMENTS}

We would like to thank all the clinicians who participated in this project.

CONFLICT OF INTEREST STATEMENT

None declared.

The results presented in this paper have not been published previously in whole or part, except in abstract format.

DATA AVAILABILITY STATEMENT

The relevant data underlying this article are available in the article. 


\section{REFERENCES:}

1. Hill, N.R., et al., Global Prevalence of Chronic Kidney Disease - A Systematic Review and MetaAnalysis. PLoS One, 2016. 11(7): p. e0158765.

2. Report:, U.R.D.S.A.D., E.o.K.D.i.t. United, and S. https://www.usrds.org/2019/view/USRDS 2019 ES final.pdf.

3. Zandecki, L., et al., Associations of changes in patient characteristics and management with decrease in mortality rates of men and women with ST-elevation myocardial infarction - a propensity score-matched analysis. Arch Med Sci, 2020. 16(4): p. 772-780.

4. Li, Y., et al., Pregnancy-associated plasma protein $A$ as a predictor of all-cause mortality and cardiovascular events in patients with chronic kidney disease: a meta-analysis of prospective studies. Arch Med Sci, 2020. 16(1): p. 8-15.

5. Wilhelm-Leen, E.R., et al., Frailty and chronic kidney disease: the Third National Health and Nutrition Evaluation Survey. Am J Med, 2009. 122(7): p. 664-71.e2.

6. Nixon, A.C., et al., Frailty and chronic kidney disease: current evidence and continuing uncertainties. Clin Kidney J, 2018. 11(2): p. 236-245.

7. Lee, S.J., H. Son, and S.K. Shin, Influence of frailty on health-related quality of life in pre-dialysis patients with chronic kidney disease in Korea: a cross-sectional study. Health Qual Life Outcomes, 2015. 13: p. 70.

8. Piątek, Ł., et al., Gender-related disparities in the treatment and outcomes in patients with nonST-segment elevation myocardial infarction: results from the Polish Registry of Acute Coronary Syndromes (PL-ACS) in the years 2012-2014. Arch Med Sci, 2020. 16(4): p. 781-788.

9. O'Hare, A.M., et al., Age affects outcomes in chronic kidney disease. J Am Soc Nephrol, 2007. 18(10): p. 2758-65.

10. Farrington, K., et al., Clinical Practice Guideline on management of older patients with chronic kidney disease stage $3 b$ or higher (eGFR<45 mL/min/1.73 m2): a summary document from the European Renal Best Practice Group. Nephrol Dial Transplant, 2017. 32(1): p. 9-16.

11. Tangri, N., et al., A predictive model for progression of chronic kidney disease to kidney failure. Jama, 2011. 305(15): p. 1553-9.

12. Bansal, N., et al., Development and validation of a model to predict 5-year risk of death without ESRD among older adults with CKD. Clin J Am Soc Nephrol, 2015. 10(3): p. 363-71.

13. Levey, A.S. and L.A. Stevens, Estimating GFR using the CKD Epidemiology Collaboration (CKD-EPI) creatinine equation: more accurate GFR estimates, lower CKD prevalence estimates, and better risk predictions. Am J Kidney Dis, 2010. 55(4): p. 622-7.

14. Harris, T.B., et al., Waist circumference and sagittal diameter reflect total body fat better than visceral fat in older men and women. The Health, Aging and Body Composition Study. Ann N Y Acad Sci, 2000. 904: p. 462-73.

15. Tangri, N., et al., Multinational Assessment of Accuracy of Equations for Predicting Risk of Kidney Failure: A Meta-analysis. Jama, 2016. 315(2): p. 164-74.

16. Ramer, S.J., et al., Health Outcome Priorities of Older Adults with Advanced CKD and Concordance with Their Nephrology Providers' Perceptions. J Am Soc Nephrol, 2018. 29(12): p. 2870-2878.

17. Hallan, S.I., et al., Implementing the European Renal Best Practice Guidelines suggests that prediction equations work well to differentiate risk of end-stage renal disease vs. death in older patients with low estimated glomerular filtration rate. Kidney Int, 2019. 96(3): p. 728-737.

18. Xiong, J., et al., Acute kidney injury in critically ill cirrhotic patients with spontaneous bacterial peritonitis: a comparison of KDIGO and ICA criteria. Arch Med Sci, 2020. 16(3): p. 569-576. 
19. Prakash, S. and A.M. O'Hare, Interaction of aging and chronic kidney disease. Semin Nephrol, 2009. 29(5): p. 497-503.

20. Ren, Q. and Q. Shi, Quality of life, symptoms, and sleep quality of elderly with end-stage renal disease receiving conservative management: a systematic review. 2019. 17(1): p. 78.

21. Ladin, K., et al., Discussing Conservative Management With Older Patients With CKD: An Interview Study of Nephrologists. Am J Kidney Dis, 2018. 71(5): p. 627-635.

22. Aguayo, G.A., et al., Agreement Between 35 Published Frailty Scores in the General Population. Am J Epidemiol, 2017. 186(4): p. 420-434. 
Table I - Baseline characteristics of study participants

\begin{tabular}{|c|c|c|c|c|c|c|c|}
\hline & & \multicolumn{4}{|c|}{ The predicted trajectory in terms of reaching ESRD/death } & \multicolumn{2}{|c|}{$\begin{array}{c}\text { The real-life trajectory in terms } \\
\text { of reaching ESRD/death }\end{array}$} \\
\hline & $\begin{array}{c}\text { Total } \\
\text { population } \\
(\mathrm{N}=958)\end{array}$ & $\begin{array}{l}\text { High risk of } \\
\text { mortality } \\
(\mathrm{N} 1=548)\end{array}$ & $\begin{array}{l}\text { Low risk of } \\
\text { mortality } \\
(\mathrm{N} 2=410)\end{array}$ & $\begin{array}{l}\text { High risk of } \\
\text { progression } \\
(\mathrm{N} 2 \mathrm{a}=101)\end{array}$ & $\begin{array}{l}\text { Low risk of } \\
\text { progression } \\
(\mathrm{N} 2 b=309)\end{array}$ & $\begin{array}{l}\text { Deaths } \\
\text { (164) }\end{array}$ & $\begin{array}{c}\text { Dialysis } \\
\text { (31) }\end{array}$ \\
\hline $\begin{array}{l}\text { Age, } \\
\text { years }\end{array}$ & $74.3( \pm 6.9)$ & $76.4( \pm 7.3)$ & $71.5( \pm 5.2)$ & $69.4( \pm 4)$ & $72.1( \pm 5.4)$ & $73.7( \pm 6.5)$ & $75.3( \pm 7.2)$ \\
\hline $\begin{array}{l}\text { Male, } \\
\text { n }(\%)\end{array}$ & $473(49.4 \%)$ & $327(59.7 \%)$ & $146(35.6 \%)$ & $43(42.6 \%)$ & $103(33.3 \%)$ & $91(55.5 \%)$ & $15(48.4 \%)$ \\
\hline $\begin{array}{l}\text { DM, } \\
\text { n }(\%)\end{array}$ & $454(47.4 \%)$ & $293(53.5 \%)$ & $161(39.3 \%)$ & $43(42.5 \%)$ & $118(38.2 \%)$ & $77(47 \%)$ & $13(41.9 \%)$ \\
\hline $\begin{array}{c}\text { HF, } \\
\text { n }(\%)\end{array}$ & $326(34 \%)$ & $281(51.3 \%)$ & $45(11 \%)$ & $4(4 \%)$ & $41(13.3 \%)$ & $46(28.1 \%)$ & $9(29.1 \%)$ \\
\hline $\begin{array}{l}\text { Stroke, } \\
\text { n }(\%)\end{array}$ & $108(11.2 \%)$ & $85(15.5 \%)$ & $23(5.6 \%)$ & $5(5 \%)$ & $18(5.8 \%)$ & $17(10.3 \%)$ & $6(19.3 \%)$ \\
\hline
\end{tabular}

ESRD, end-stage renal disease; DM, diabetes mellitus; HF, heart failure.

Data are mean $( \pm \mathrm{SD})$, frequencies (or percentages). 


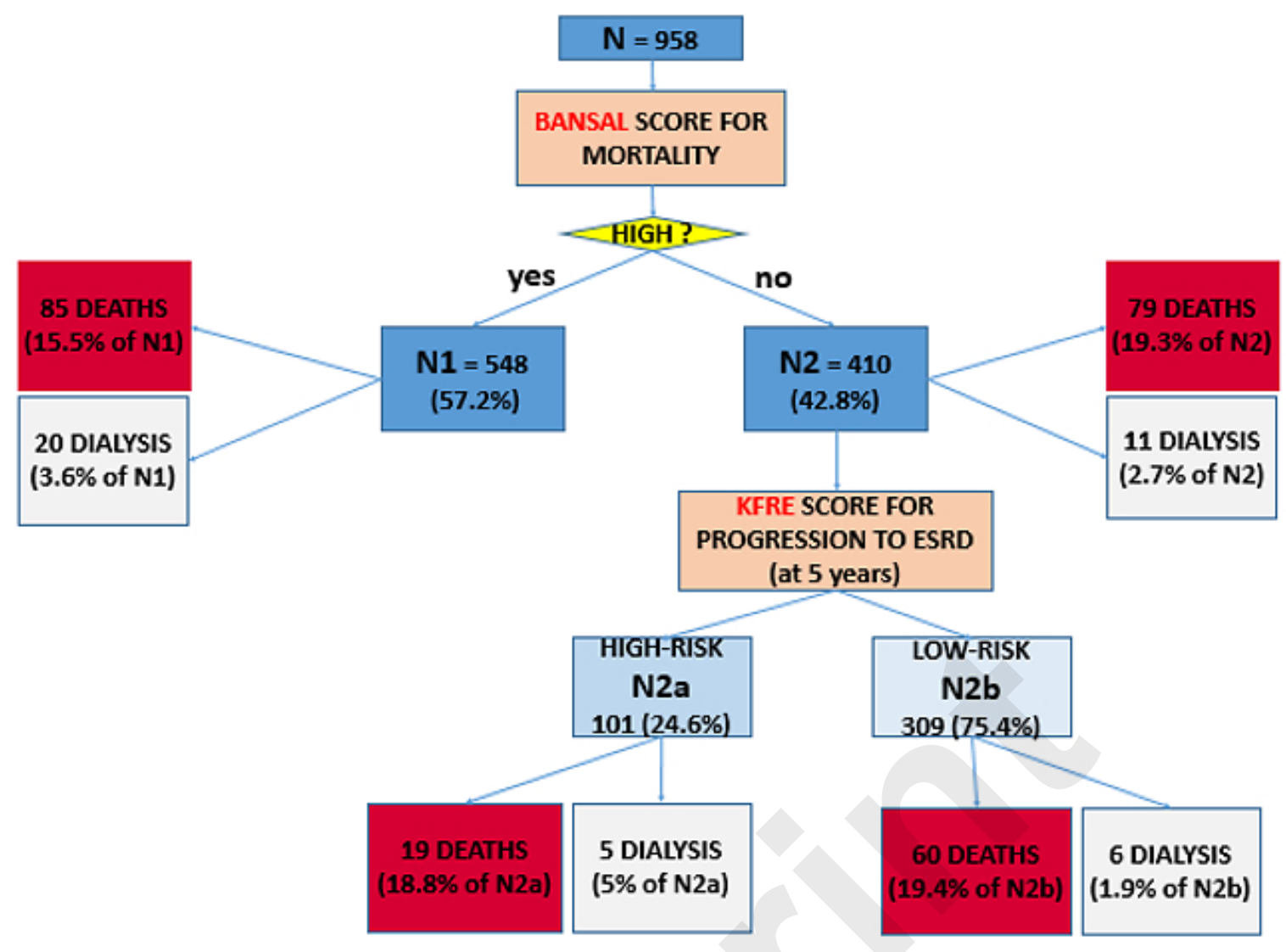

Flowchart 1: The real-life trajectory of the study population in terms of reaching dialysis/death. 